Olatunji, B. O. \& Broman-Fulks, J. J. (2007). A taxometric study of the latent structure of disgust sensitivity: Converging evidence for dimensionality. Psychological Assessment, 19(4): 437-448. Published by the American Psychological Association (ISSN: 1939-134X).

\title{
A taxometric study of the latent structure of disgust sensitivity: Converging evidence for dimensionality
}

\author{
Brunmi O. Olatunji and Joshua J. Broman-Fulks
}

\begin{abstract}
Disgust sensitivity has recently been implicated as a specific vulnerability factor for several anxiety-related disorders. However, it is not clear whether disgust sensitivity is a dimensional or categorical phenomenon. The present study examined the latent structure of disgust by applying three taxometric procedures (maximum eigenvalue, mean above minus below a cut, and latent-mode factor analysis) to data collected from 2 large nonclinical samples on 2 different measures of disgust sensitivity. Disgust sensitivity in the first sample $(n=1,153)$ was operationalized by disgust reactions to food, animals, body products, sex, body envelope violations, death, hygiene, and sympathetic magic, as assessed by the Disgust Sensitivity Scale (J. Haidt, C. McCauley, \& P. Rozin, 1994). Disgust Sensitivity Scale indicators of core, animal reminder, and contamination disgust were also examined in the 1st sample. Disgust sensitivity in the 2 nd independent sample $(n=1,318)$ was operationalized by disgust reactions to animals, injections and blood draws, mutilation and death, rotting foods, and odors, as assessed by the Disgust Emotion Scale (R. A. Kleinknecht, E. E. Kleinknecht, \& R. M. Thorndike, 1997). Results across both samples provide converging evidence that disgust sensitivity is best conceptualized as a dimensional construct, present to a greater or lesser extent in all individuals. These findings are discussed in relation to the conceptualization and assessment of disgust sensitivity as a specific dimensional vulnerability for certain anxiety and related disorders.
\end{abstract}


Disgust sensitivity (DS), defined as a predisposition toward experiencing disgust (de Jong \& Merckelbach, 1998; Olatunji \& Sawchuk, 2005), has been described as a genetically based personality trait that operates as a specific vulnerability factor for certain disorders (Muris, 2006). Indeed, numerous descriptive and experimental studies have now implicated DS in the development of spider phobia (de Jong \& Merckelbach, 1998; de Jong \& Muris, 2002; de Jong, Peters, \& Vanderhallen, 2002; Merckelbach, de Jong, Arntz, \& Schouten, 1993; Mulkens, de Jong, \& Merckelbach, 1996; Sawchuk, Lohr, Tolin, Lee, \& Kleinknecht, 2000; Tolin, Lohr, Sawchuk, \& Lee, 1997; Woody, McLean, \& Klassen, 2005) and blood-injection-injury (BII) phobia (de Jong \& Merckelbach, 1998; Olatunji, Arrindell, \& Lohr, 2005; Olatunji, Sawchuk, de Jong, \& Lohr, 2006; Olatunji, Williams, Sawchuk, \& Lohr, 2006; Page, 2003; Sawchuk, Lohr, Lee, \& Tolin, 1999; Sawchuk, Lohr, Westendorf, Meunier, \& Tolin, 2002; Tolin et al., 1997). Although it has been suggested that the relation between DS and specific phobias is illusory and mediated by negative affect (i.e., Thorpe \& Salkovskis, 1998), recent studies have indicated that DS possesses a specific relation with spider phobia and BII phobia that is not accounted for by negative affect and therefore may constitute a specific risk factor for these phobic conditions (Muris, Merckelbach, Schmidt, \& Tierney, 1999; Olatunji, 2006; Olatunji, Sawchuk, Lohr, \& Patten, in press).

Theoretical accounts consider DS as a personality trait that functions to facilitate avoidance of stimuli that may communicate uncleanliness, contamination, and the potential for disease (Matchett \& Davey, 1991; Olatunji \& Sawchuk 2005; Woody \& Teachman, 2000). Accordingly, research findings have begun to also implicate DS in the etiology of contamination-based obsessive-compulsive disorder (OCD; Mancini, Gragnani, \& D'Olimpio, 2001; Olatunji, Sawchuk, Lohr, \& de Jong, 2004; Olatunji, Tolin, Huppert, \& Lohr, 2005; Schienle, Stark, Walter, \& Vaitl, 2003; Thorpe, Patel, \& Simonds, 2003; Woody \& Tolin, 2002). In fact, recent neuroimaging studies suggest that the neurocircuits (e.g., the anterior insular cortex) involved in DS are relevant to OCD and, in particular, contamination obsessions and washing compulsions (Husted, Shapira, \& Goodman, 2006). The relation between DS and contamination-based OCD also appears to be unique and unaccounted for by symptoms of fear, anxiety, and depression (Olatunji, Lohr, Sawchuk, \& Tolin, 2007; Olatunji, Sawchuk, Arrindell, \& Lohr, 2005; Tolin, Woods, \& Abramowitz, 2006). Research on the role of DS in OCD has recently been extended to health anxiety and hypochondriasis. For example, Davey and Bond (2006) found highly significant correlations between DS and measures of hypochondriasis and health anxiety, even when levels of trait anxiety were controlled for.

DS also appears to play a role in the development and maintenance of eating disorders, given that several derivatives of disgust (e.g., shame) are commonly associated with foods (Phillips, Senior, Fahy, \& David, 1998; Rozin \& Fallon, 1987; Troop, Murphy, Bramon, \& Treasure, 2000). Although empirical studies investigating the proposed association between DS and eating disorders have been limited, interesting findings have been generated from this sparse literature. First, measures of DS correlate positively with eating disorder symptoms in women (Davey, Buckland, Tantow, \& Dallos, 1998). Furthermore, when researchers compared samples with and without eating disorders, significant group differences emerge on DS toward food, the physical body, and bodily products (Davey et al., 1998; Troop et al., 2000; Troop, Treasure, \& Serpell, 2002). Finally, women in remission from an eating disorder showed significant 
reductions in bodily focused DS in comparison to women who continued to meet diagnostic criteria (Troop et al., 2002).

The current literature suggests that DS may play an etiological role in anxiety-related disorders that involve the protection of the self from physical and/or psychological contamination (Olatunji \& Sawchuk, 2005). In many ways, the notion that DS operates as a specific vulnerability for certain anxiety disorders parallels the growing body of literature implicating anxiety sensitivity (the fear of anxiety and anxiety-related sensations; Reiss \& McNally, 1985) as a risk factor for the development of panic disorder and other anxiety pathology (McNally, 2002). Like anxiety sensitivity and many other personality risk factors, DS has traditionally been conceptualized as an individual-differences variable that exists in all persons, varying between individuals by degree rather than kind. To date, researchers have operated from the assumption that the latent structure of DS is dimensional (Haidt, McCauley, \& Rozin, 1994), and theoretical (Rozin, Haidt, $\&$ McCauley, 2000) and empirical work have provided supportive evidence that DS is, indeed, a multifaceted construct (Olatunji, Williams, Lohr, \& Sawchuk, 2005).

To our knowledge, researchers have yet to directly test the accuracy of the assumption that DS is continuous. There are theoretical reasons why DS may be categorical rather than continuous. For example, the experience of disgust is adaptive in that it functions to prevent infection and disease (Olatunji et al., 2004). Sensitivity to disgust-related cues may then serve to warn the organism to internal and external sources of contamination. It remains unclear how an evolutionarily adaptive process, normally distributed in the general population, comes to operate as risk factors for specific disorders (Berstein et al., 2007). One possibility is that aversive learning experiences or certain gene-environmental interactions may qualitatively change the adaptive function of DS to become dysfunctional, consequently facilitating the developmental separation of DS into unique and distinct categories. It may then be theoretically and practically useful to conceptualize one category of DS as a normative form of DS and the other as a vulnerability-conferring form of DS (i.e., conferring risk for specific disorders). Indeed, recent basic laboratory research has shown that once associative learning of disgust occurs, it is often highly resistant to extinction (Olatunji, Forsyth, \& Cherian, 2007). This resistance to extinction may thereby facilitate and maintain the taxonic split between adaptive and maladaptive latent forms of DS. The maladaptive latent form of DS may then be viewed as a highly potent agent that is necessary but may not be sufficient for causing a certain psychological disorder (e.g., contamination-based OCD).

There are also empirical reasons why DS may be categorical rather than continuous. Although not without debate, there is some recent evidence that anxiety sensitivity (a risk factor for panic disorder and other anxiety disorders), a related construct that has been shown to interact with DS in predicting symptoms of contamination-based OCD (Cisler, Reardon, Williams, \& Lohr, 2007), may be categorical (Bernstein et al., 2007). Furthermore, using hierarchical agglomerative cluster analysis to categorize disgust-evoking stimuli, Marzillier and Davey (2004) found three distinct clusters, corresponding to (a) nondisgust or "disgust-irrelevant" items; (b) "primary" disgust items, which included a range of disgust-relevant items characterized by their ability to elicit fear of oral incorporation and of one's animal origin; and (c) "complex" disgusts, consisting mainly of behaviors or activities that are considered to be socially or morally 
unacceptable. Although cluster analysis is a limited method of detecting latent discontinuity, the identification of disgust clusters may be viewed as indirect evidence for a taxonic model of disgust. Moreover, some researchers have elected to dichotomize DS by means of arbitrary cutoff scores (e.g., individuals scoring in the upper third and the lower third on a measure of DS), treating DS, at least on the level of manifest measurement, as a categorical variable (e.g., Schienle, Stark, \& Vaitl, 2001). The simultaneous measurement of DS as a continuous and dichotomous construct in the current literature raises questions as to whether the latent structure of DS is categorical (i.e., taxonic) or dimensional.

A taxon is a nonarbitrary latent category whose members are qualitatively distinct from nonmembers (Haslam, 2003). Nature and society are known to contain numerous taxa, including chemical elements, biological species, and occupational categories. In contrast, nontaxonic phenomena, such as age, temperature, or height, are characterized by continuous or dimensional latent structure. Taxometrics is a branch of applied mathematics designed specifically to discern the latent structure (i.e., categorical vs. continuous distribution) of phenomena by evaluating whether the numerical relations among various indicators of a conjectured taxon fit a pattern consistent with a naturally occurring class or a continuous dimension (Meehl \& Golden, 1982; Waller \& Meehl, 1998). Taxometric procedures have a long history in the literature (e.g., Meehl, 1973) and have been used effectively to identify the latent structure of numerous clinical phenomena, including both dimensional (e.g., posttraumatic stress reactions; Broman-Fulks et al., 2006) and taxonic constructs (e.g., schizotypy; Lenzenweger \& Korfine, 1992). However, these procedures have only recently been applied to the study of the latent structure of anxiety disorder risk factors. For example, there is now evidence that worry, a risk factor for and core symptom of generalized anxiety disorder, is dimensional (A. M. Ruscio, Borkovec, \& Ruscio, 2001). In contrast, the findings of several recent studies of anxiety sensitivity have described this construct as a categorical, or taxonic, clinical syndrome (e.g., Bernstein, Zvolensky, Kotov, et al., 2006; Bernstein, Zvolensky, Stewart, Comeau, \& Leen-Feldner, 2006; Bernstein, Zvolensky, Weems, Stickle, \& Leen-Feldner, 2005; Schmidt, Kotov, Lerew, Joiner, \& Ialongo, 2005). Thus, there appear to be both categorical and continuous risk factors for the development of anxiety disorders.

Although DS has been gaining increased recognition as a genetically based risk factor for certain phobias, contamination-based OCD, and perhaps even health anxiety-hypochondriasis and eating disorders (e.g., Muris, 2006; Olatunji \& Sawchuk, 2005), the latent structure of DS remains unclear. If DS is, indeed, a risk factor for such anxiety-related conditions, it is of critical importance to extend work on the underlying structure of this construct beyond factor and cluster analytic investigations. Research examining the latent structure of DS could advance knowledge relating to the selection of measures that are consistent with the true latent structure of DS (i.e., disperse scores broadly and discriminate effectively in all areas of the DS dimension vs. sorting individuals at an optimal level in DS in an attempt to maximize valid classifications). Identifying the latent structure of DS may also help to inform etiological models. Specifically, taxonic latent structure is suggestive of a discrete etiological source, such as a particular gene, brain disorder, environmental stressor, or particular interaction of such variables. In contrast, dimensional structure implies an additive or graded etiology (i.e., it is multiply determined). Thus, with regard to DS, a dimensional latent structure would suggest that various 
environmental-related and person-specific factors (e.g., conditioning event, behavioral inhibition) may be important in determining the etiology of DS.

The overarching goal of the present study was to examine the latent structure of DS using a series of taxometric procedures. Although the latent structures of various fear- and anxietyrelated phenomena have been examined in the research literature (e.g., Bernstein, Zvolensky, Kotov, et al., 2006; Kotov, Schmidt, Lerew, \& Joiner, 2005), with both taxonic and dimensional findings, a similar analysis has yet to be extended to indexes of disgust. There is some suggestive evidence from factor analytic studies that DS is a multifaceted construct that may be best conceptualized as a dimensional construct (Haidt et al., 1994; Olatunji, Williams, et al., 2005). Therefore, it was hypothesized that multiple taxometric procedures would yield a continuous solution of DS across two large independent samples using two different measures of the DS construct.

\section{METHOD}

\section{Participants}

Participants consisted of two large independent samples of undergraduate students. Participants in the first sample, who completed the Disgust Sensitivity Scale (DSS; Haidt et al., 1994) were 1,153 male $(n=264)$ and female $(n=859)$ undergraduates recruited from two large southern universities. DSS completers ranged in age from 18 to $52(M=19.25, S D=2.55)$. The second independent sample of participants completed the Disgust Emotion Scale (DES; Kleinknecht, Kleinknecht, \& Thorndike, 1997) and consisted of 1,318 undergraduates from a large southern university. DES completers consisted predominately of women $(n=884,86 \%)$ and ranged in age from 18 to $52(M=20.67, S D=4.78)$. Participants received course credit in exchange for their participation.

\section{Measure}

The DSS (Haidt et al., 1994) assesses sensitivity to seven domains of potential disgust-eliciting stimuli (i.e., food; animals; body products; sex; body envelope violations, e.g., body piercing; death; and hygiene) and levels of sympathetic magic (i.e., beliefs about the transmission of contagion). On the basis of a well-articulated and empirically supported theory of disgust (Rozin \& Fallon, 1987; Rozin et al., 2000), the eight subscales of the DSS can be divided into three theoretically meaningful and more reliable domains of disgust. Core disgust, consisting of the food, animals, and body products subscales, serves primarily as a food-rejection response and centers on the avoidance or oral incorporation of disgusting stimuli. Animal reminder disgust, consisting of the sex, body envelope violations, and death subscales, reflects stimuli that remind us of our animalness and our animal origins. Last, interpersonal or contamination disgust, consisting of the hygiene and sympathetic magic subscales, is marked by strangeness, disease, and contagion potency. Prior research has provided evidence for the validity of this classification of the eight subscales of the DSS. For example, de Jong and Merckelbach (1998) found that 
core disgust was specifically related to spider fear, whereas animal reminder was specifically related to BII fears. The 32-item DSS includes 16 true-false items and 16 items that are rated on a 3-point scale. The 32 items assess the extent to which participants find the experience not disgusting at all, slightly disgusting, or very disgusting. Three of the true-false items are reverse scored. Each of the eight subscales of the DSS contains 4 items: 2 true-false items and 2 Likert-type items. Haidt et al. (1994) suggested that researchers may calculate a total score for overall DS by summing responses to the 32 items. Internal consistency of the DSS ranges between .80 and .87 (Haidt et al., 1994).

The DES (Kleinknecht et al., 1997) is a 30-item scale measuring five domains of DS: animals, injections and blood draws, mutilation and death, rotting foods, and odors. Participants are asked to rate their degree of disgust or repugnance if they were to be exposed to each item, using a 5-point Likert scale ranging from $0=$ no disgust or repugnance at all to $4=$ extreme disgust or repugnance. One may calculate a total score for DS by summing responses to the 30 items. Olatunji, Sawchuck, de Jong, and Lohr (2007) provided evidence for the psychometric properties of the DES in two independent studies of nonclinical participants. In their study, the total DES score demonstrated excellent internal consistency $(\alpha=.91)$. Studies have also presented supportive evidence for the convergent validity of the DES (e.g., Kleinknecht et al., 1997).

\section{Procedure}

Sample 1 participants completed the DSS via an Internet site or in groups of 8-12 individuals as part of a larger questionnaire battery. Sample 2 participants completed the DES in groups of 510 individuals as part of a larger questionnaire battery.

\section{Data Analytic Strategy}

Instead of traditional statistical significance testing, a multiple hurdles consistency testing approach is recommended as part of the taxometric method to protect against spurious results (Meehl, 1995; Waller \& Meehl, 1998). If a latent taxon exists, the results of multiple independent taxometric procedures should converge in support of a taxon. In accordance with this recommendation, the present study used three independent taxometric procedures-maximum eigenvalue (MAXEIG), mean above minus below a cut (MAMBAC), and latent-mode factor analysis (L-Mode) - to analyze the latent structure of DS as measured by two well-established measures: the DSS and the DES. Following is a brief explanation of the mathematical rationale behind each taxometric procedure. 


\section{MAXEIG}

MAXEIG (Waller \& Meehl, 1998) is a multivariate extension of the frequently used maximum covariance procedure (Meehl \& Yonce, 1996). MAXEIG calculates and plots eigenvalues from all remaining indicators across successive intervals of an input indicator. Each indicator serves as the input indicator on one analysis, resulting in one MAXEIG plot per indicator. If a taxon is present, data typically yield plots with peaked curves. In contrast, dimensional data tend to produce relatively flat plots.

\section{MAMBAC}

The MAMBAC (Meehl \& Yonce, 1994) procedure is based on the assumption that if two discrete groups exist (i.e., taxon and nontaxon), mean differences between groups will occur on valid indicators of group membership. When mean differences are plotted as a function of one variable, taxonic constructs tend to produce peaked plots at the point that best differentiates the two groups, whereas dimensional constructs tend to produce relatively flat or bowl-shaped plots.

\section{L-Mode}

L-mode (Waller \& Meehl, 1998) involves a factor analysis on all candidate indicators and plots the distribution of scores on the first principal factor. If the construct under investigation is taxonic, a bimodal distribution of factor scores will be evident. In contrast, if the variable is dimensional, factor scores will be unimodally distributed.

The algorithms used to perform the MAXEIG, MAMBAC, and L-Mode analyses were obtained from J. Ruscio (2006). One advantage of the Ruscio programs is that they simultaneously create simulated taxonic and dimensional data plots based on data that have similar distributional characteristics (e.g., skew, kurtosis, sample size) to the empirical data (J. Ruscio, Haslam, \& Ruscio, 2006; J. Ruscio, Ruscio, \& Meron, 2007). One can then use the simulations to aid in the interpretation of the empirical data plots by comparing the experimental data plots with the simulated dimensional and taxonic plots. Simulations were created with algorithms detailed in J. Ruscio (2007). Simulated dimensional data were created via an iterative procedure that matches the exact distributions of all indicators, including their skew, kurtosis, and discrete values. In contrast, we generated simulated taxonic data sets by employing a fallible criterion variable, denoting the putative class membership of each case, to isolate taxon and complement distributions. The same iterative procedure was then employed to create simulated taxon and complement distributions, which were merged to produce the simulated data set for the taxonic case. In the present study, cases were sorted into putative taxon and nontaxon membership according to Bayes's theorem in MAXEIG, the grand mean base rate estimate in MAMBAC, and the nearest mode in L-Mode. Although some researchers have voiced concerns over the use of sample-specific simulations and the programs that generate them (Beach, Amir, $\& \mathrm{Bau}, 2005)$, a recent reanalysis of the data presented evidence to support their use in taxometric research (J. Ruscio \& Marcus, 2007). 


\section{Indicator Selection}

Due to the large volume of analyses that would be involved if each of the 32 DSS items and 30 DES items were used as individual indicators, composite indicators were derived from the DSS and DES based on prior factor analytic studies and empirically supported theoretical models (Björklund \& Hursti, 2004; Olatunji, Sawchuk, de Jong, \& Lohr, 2007; Rozin et al., 2000). Specifically, research has indicated that the DSS assesses DS across eight categories (food, animals, body products, sex, body envelope violations, death, hygiene, and sympathetic magic), which can be further divided into three domains (core, animal reminder, and contamination disgust), whereas the DES measures DS across five categories (animals, injections and blood draws, mutilation and death, rotting foods, and odors). Items loading on each of these categories or domains were summed to create three sets of indicators representing the eight DSS subfactors (DSS-8; see Table 1 for descriptive statistics), three DSS domains (DSS-3; see Table 2 for descriptive statistics), and five DES subfactors (see Table 3 for descriptive statistics). The creation of summed indicators has the advantage of yielding more reliable indicators than would be obtained if individual item responses were used as indicators. Summation of the items loading on each of the DSS-8 subscales created indicators with an 8point range (i.e., 0-4 with 0.5 intervals), whereas summation of the DSS-3 items produced indicators with 0- to 24-point ranges, depending on the subscale. Summation of the DES items generated indicators with a range of 24.

Table 1

Descriptive Statistics, Reliability, and Pearson Correlation Coefficients for the Eight Indicators of the Disgust Sensitivity Scale

\begin{tabular}{lccccccccc}
\hline \multicolumn{1}{c}{ Indicator } & $M(S D)$ & Range & 1 & 2 & 3 & 4 & 5 & 6 & 7 \\
\hline 1. Food & $1.98(0.94)$ & $0.00-4.00$ & .37 & & & & & & \\
2. Animals & $2.51(1.06)$ & $0.00-4.00$ & .43 & .54 & & & & & \\
3. Body products & $2.36(1.01)$ & $0.00-4.00$ & .35 & .41 & .47 & & & & \\
4. Sex & $3.07(0.82)$ & $0.00-4.00$ & .22 & .16 & .19 & .46 & & & \\
5. Envelope violations & $2.38(1.18)$ & $0.00-4.00$ & .32 & .39 & .36 & .11 & .66 & & \\
6. Death & $1.88(1.23)$ & $0.00-4.00$ & .35 & .39 & .33 & .19 & .54 & .65 & .25 \\
7. Hygiene & $1.57(0.96)$ & $0.00-4.00$ & .28 & .26 & .28 & .23 & .13 & .48 \\
8. Sympathetic magic & $1.98(1.03)$ & $0.00-4.00$ & .38 & .37 & .36 & .27 & .32 & .42 & .42 \\
\hline
\end{tabular}

Note. All correlation coefficients are significant at $p<.001$. Cronbach's alpha for each subscale is provided on the diagonal.

Table 2

Descriptive Statistics, Reliabilities, and Pearson Correlation Coefficients for the Three Theoretically Derived Indicators of the Disgust Sensitivity Scale

\begin{tabular}{lccccr}
\hline \multicolumn{1}{c}{ Indicator } & $M(S D)$ & Range & 1 & 2 & 3 \\
\hline 1. Core disgust & $6.87(2.33)$ & $0.00-12.00$ & .70 & & \\
2. Animal reminder disgust & $7.33(2.40)$ & $0.00-12.00$ & .54 & .73 & \\
3. Contamination disgust & $3.56(1.68)$ & $0.0-8.00$ & .49 & .44 & .63 \\
\hline
\end{tabular}

Note. All correlation coefficients are significant at $p<, 001$. Cronbach's alpha for each indicator is provided on the diagonal. 
Table 3

Descriptive Statistics, Reliabilities, and Pearson Correlation Coefficients for the Five Indicators of the Disgust Emotion Scale

\begin{tabular}{|c|c|c|c|c|c|c|c|}
\hline Indicator & $M(S D)$ & Range & 1 & 2 & 3 & 4 & 5 \\
\hline 1. Animals & $5.68(4.60)$ & $0.00-24.00$ & .80 & & & & \\
\hline 2. Injections and blood draws & $5.96(5.62)$ & $0.00-24.00$ & .44 & .90 & & & \\
\hline 3. Mutilation and death & $11.58(5.67)$ & $0.00-24.00$ & .55 & .55 & .87 & & \\
\hline 4. Rotting foods & $8.29(4.76)$ & $0.00-24.00$ & .54 & .32 & .43 & .88 & \\
\hline 5. Odors & $11.80(4.99)$ & $0.00-24.00$ & .55 & .37 & .54 & .67 & .89 \\
\hline
\end{tabular}

Note. All correlation coefficients are significant at $p<.001$. Cronbach's alpha for each subscale is provided on the diagonal.

It is generally recommended that indicators should be uncorrelated within the proposed taxon and complement classes (i.e., have low nuisance covariance; Meehl \& Yonce, 1994). Thus, mean within-group correlations for the DSS-8, DSS-3, and DES indicators were examined. As noted above, cases were sorted into putative taxon and nontaxon membership via Bayes's theorem in MAXEIG, the grand mean base rate estimate in MAMBAC, and the nearest mode in L-Mode. Nuisance covariances were found to be low for the DSS-8 (taxon group $=0.08$, nontaxon group $=0.06$ ), DSS-3 (taxon group $=0.11$, nontaxon group $=0.09)$, and DES (taxon group $=0.18$, nontaxon group $=0.19)$. As is preferable, indicator correlations among the total samples were substantially higher for the DSS-8 $(M=0.31)$, DSS-3 $(M=0.49)$, and DES $(M=$ 0.49). Additionally, it is generally recommended that indicators possess high degrees of validity. Previous research has found that indicator validities of one standard deviation or greater are sufficient for taxometric research (Meehl \& Golden, 1982). Indicators used in the present study were found to be valid, 1 with mean separations of 1.38, 1.76, and 1.60 standard deviations for the DSS-8, DSS-3, and DES indicators, respectively. Furthermore, skew was low among the DSS-8 ( $M=-0.11)$, DSS-3 $(M=-0.48)$, and DES $(M=0.78)$ indicators. Thus, the indicators utilized in the present research were deemed appropriate for taxometric analysis.

\section{RESULTS}

\section{Suitability Test}

Previous research has suggested that for data to be considered suitable for taxometric analysis, the plots generated from the simulated taxonic and dimensional data should be clearly distinguishable from one another (J. Ruscio et al., 2006, 2007). That is, if taxonic and dimensional data are generated to simulate the research data and the output plots from the simulations are not differentiable (i.e., both sets look taxonic or both sets appear dimensional), then it is unlikely that the data will be able to provide a clear answer regarding taxonicity. As a preliminary test of the suitability of the present research data for conducting a taxometric analysis of DS, we examined the simulated taxonic and dimensional plots generated on the basis of the research data. Results indicated that the simulated taxonic and dimensional data plots were clearly distinguishable from each other for all MAXEIG, MAMBAC, and L-Mode analyses (see Figures 1, 2, and 3). Thus, the present data passed the initial suitability test and were submitted to taxometric analysis. 

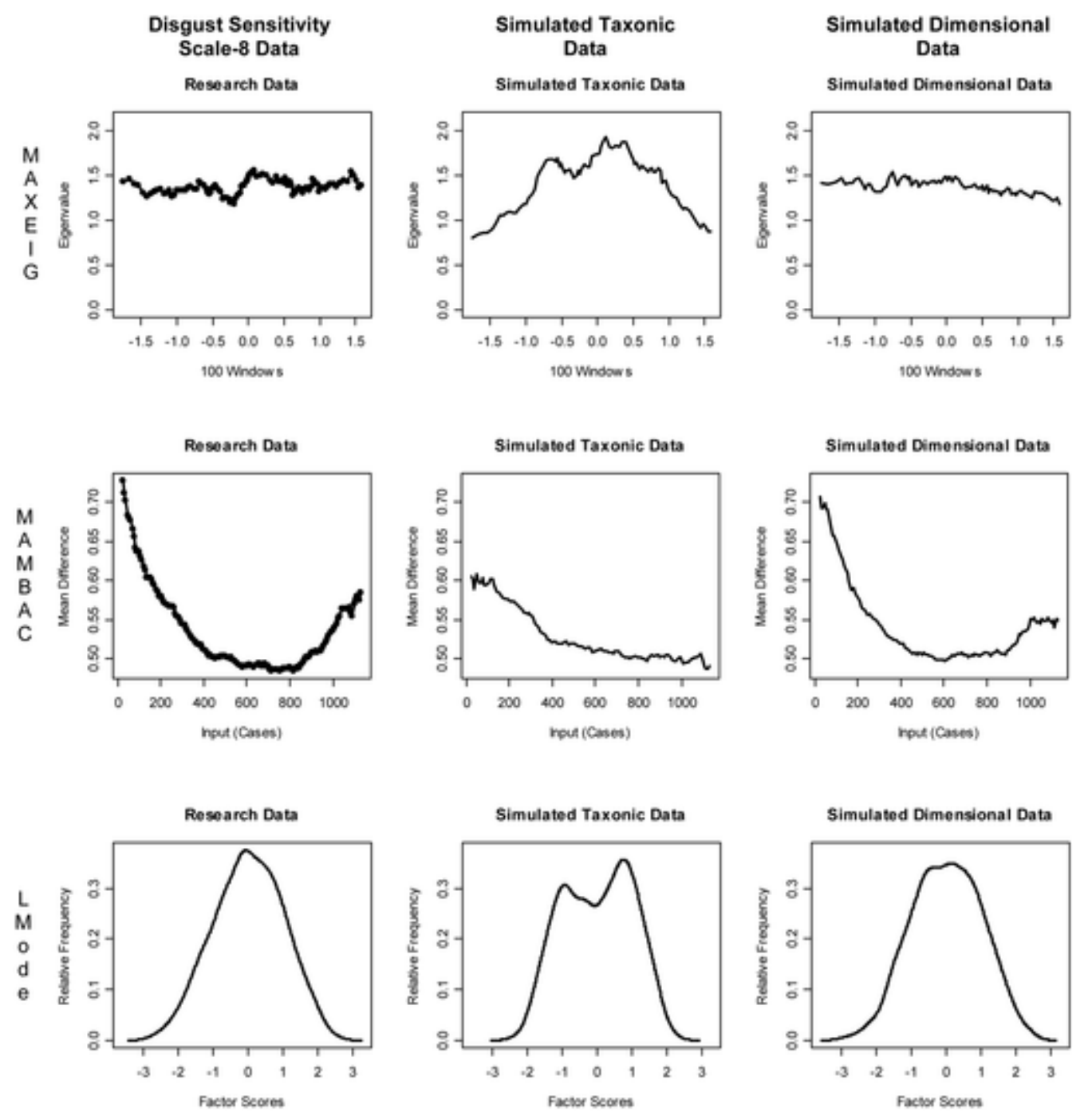

Figure 1. Averaged maximum eigenvalue (MAXEIG), mean above minus below a cut (MAMBAC), and latent-mode factor analysis (L-Mode) plots for the Disgust Sensitivity Scale-8 indicator data, simulated taxonic data, and simulated dimensional data. 

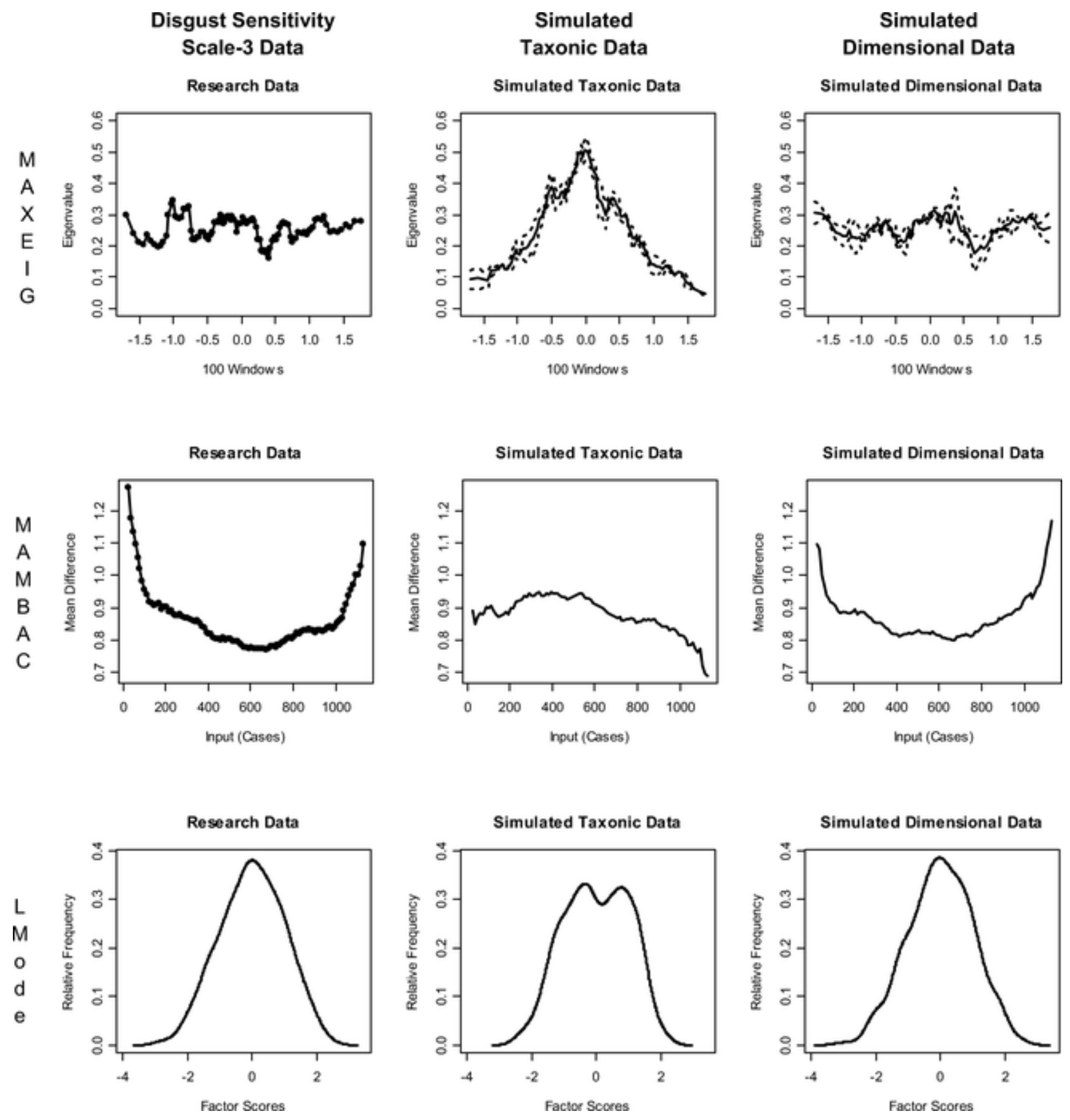

Figure 2. Averaged maximum eigenvalue (MAXEIG), mean above minus below a cut (MAMBAC), and latent-mode factor analysis (L-Mode) plots for the Disgust Sensitivity Scale-3 indicator data, simulated taxonic data, and simulated dimensional data. 

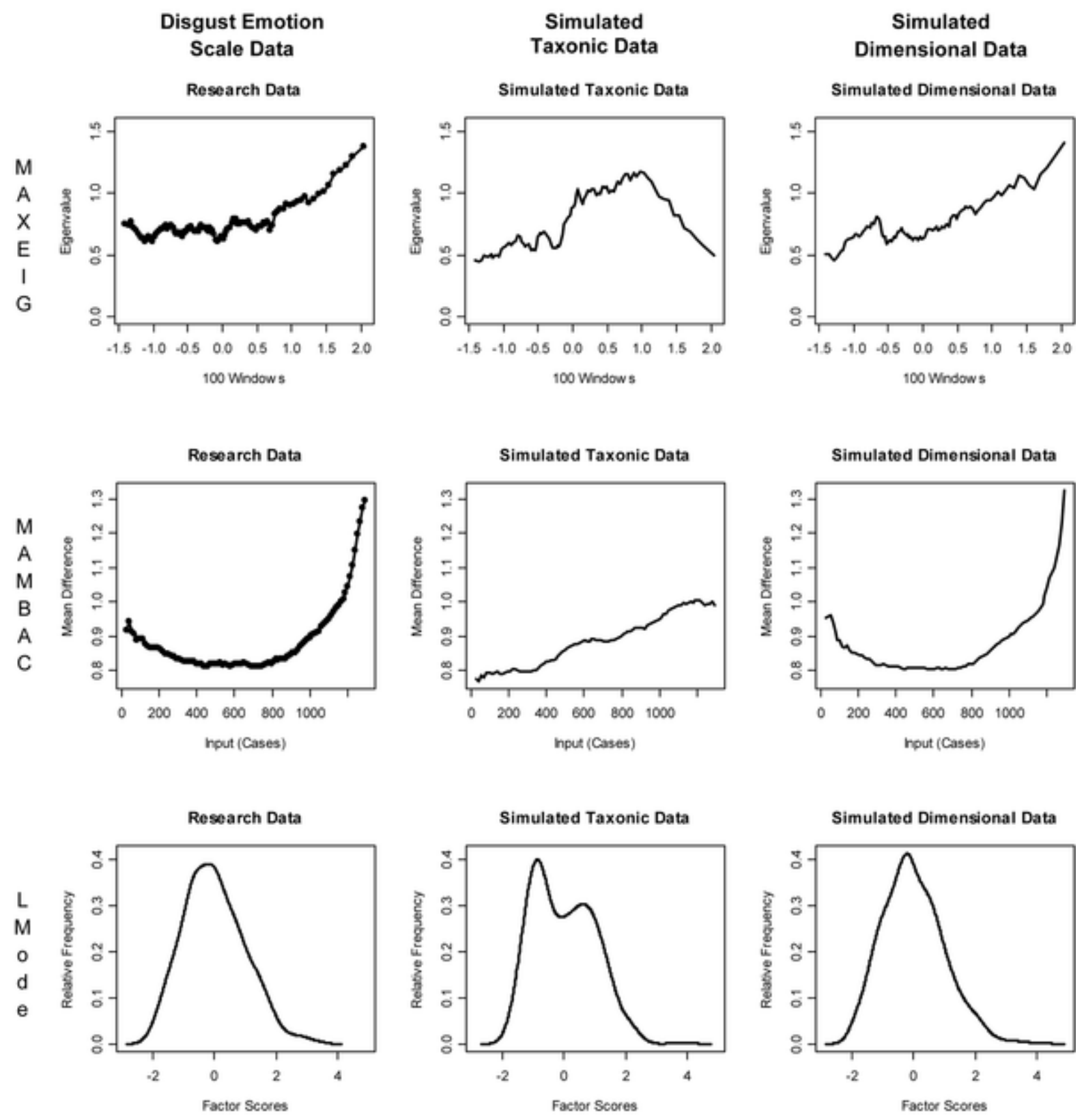

Figure 3. Averaged maximum eigenvalue (MAXEIG), mean above minus below a cut (MAMBAC), and latent-mode factor analysis (L-Mode) plots for the Disgust Emotion Scale data, simulated taxonic data, and simulated dimensional data.

\section{DSS-8 Indicator Results}

MAXEIG analyses were run first with the DSS-8 indicators. All eight of the resulting MAXEIG plots for the DSS-8 indicators failed to demonstrate any peaks that would be consistent with a taxon. Rather, all plots were relatively flat, with minor fluctuations around a mean eigenvalue, thus favoring a dimensional solution. 2 To reduce the quantity of graphical output, we present 
only the averaged plot for each taxometric procedure. Thus, the results of the MAXEIG procedure provide initial support for a dimensional latent structure underlying DS.

As noted above, taxometric procedures rely on replication of findings across independent procedures to ensure accuracy of results (Meehl, 1995). Thus, even though the MAXEIG procedure supplied evidence for a dimensional structure, MAMBAC analyses were conducted as an additional measure of consistency. The eight DSS-8 indicators produced 56 MAMBAC plots, none of which demonstrated a distinctive taxonic peak. Rather, all of the MAMBAC plots were relatively flat or demonstrated the characteristically dimensional concave shape. Thus, MAMBAC results provide further evidence for a dimensional solution for DS.

L-Mode was run as a third measure of consistency. Unlike MAXEIG and MAMBAC, L-Mode generates a single data plot regardless of the number of indicators. Results of the L-Mode procedure clearly favored a dimensional solution. Specifically, the L-Mode plot based on the empirical data demonstrated a unimodal distribution, whereas taxonic data generally evidence two distinct peaks (a bimodal distribution). Thus, the L-Mode results complement the findings of MAXEIG and MAMBAC, which, taken together, provide consistent evidence for a dimensional latent structure for DS.

\section{DSS-3 Indicator Results}

As noted above, although there is factor analytic evidence in support of the existence of the eight facets of DS (i.e., Björklund \& Hursti, 2004), theoretical formulations and empirical evidence indicate that the DSS also consists of three general domains of DS that correspond to core, animal reminder, and contamination disgust (i.e., Rozin et al., 2000). In addition to the low reliability of the DSS-8 indicators (see Table 1), which has also been reported in other studies (i.e., Haidt et al., 1994), two of the DSS-8 indicators failed to meet the minimum validity criteria. Thus, to ensure that the dimensional findings for the DSS-8 were not simply due to the psychometric properties of the indicators, we conducted separate MAXEIG, MAMBAC, and LMode analyses using indicators derived from the three-factor solution. The three DSS-3 indicators were more reliable (see Table 2) and demonstrated higher degrees of validity in comparison with the DSS-8 indicators, with all DSS-3 indicators showing separations of greater than one and a half standard deviations.

The application of the MAXEIG procedure to the DSS-3 data generated three plots, all of which were relatively flat, with minor fluctuations around a mean eigenvalue. Refer to Figure 2 for the averaged MAXEIG, MAMBAC, and L-Mode curves generated with the DSS-3 data. Similarly, the MAMBAC procedure produced six plots with the characteristically dimensional bowl shape. Furthermore, results of the L-Mode procedure revealed a clearly unimodal distribution. Thus, the collective results of the DSS-3 analyses were consistent with those of the DSS-8 data in supporting a dimensional DS solution. 


\section{DES Results}

As an additional measure of consistency, MAXEIG, MAMBAC, and L-Mode were also applied to a separate measure of DS-the DES. Five indicators derived from the DES were submitted to the MAXEIG procedure first. Results indicated five relatively flat MAXEIG plots, none of which evidenced any peaks that were typical of a taxon. See Figure 3 for the averaged MAXEIG, MAMBAC, and L-Mode curves based on the DES data. Similarly, MAMBAC analyses of the DES data generated 20 plots, none of which evidenced any peaks that were consistent with a taxon, thus providing further evidence for a dimensional solution. L-Mode was the final consistency test conducted on the DES data. The resulting factor plot revealed a single peak, which is characteristic of dimensional data. Thus, the results of MAXEIG, MAMBAC, and LMode analyses of the DES data provide convergent evidence in support of a latent DS dimension.

\section{Sample-Specific Simulations}

As noted above, simulated taxonic and dimensional data with similar distributional characteristics to the research data can be generated compared with the results produced by the research data. As can be seen in Figures 1-3, the averaged MAXEIG, MAMBAC, and LMode curves generated by the DSS-8, DSS-3, and DES data closely resembled the simulated dimensional plots. In contrast, the simulated MAXEIG plots generated distinct peaks that were absent in the data plots. Furthermore, the simulated taxonic plots produced by MAMBAC showed cusps, whereas the taxonic L-Mode plots demonstrated clear bimodal distributions that were also missing from the empirical data and simulated dimensional plots. Thus, the samplespecific simulations provide additional support for a dimensional DS structure.

\section{Base Rate Estimates}

In addition to producing plots that can be visually examined for latent structure, each MAXEIG, MAMBAC, and L-Mode analysis also estimates the base rate of the proposed taxon (if one exists). Base rate estimates generated by each MAXEIG, MAMBAC, and L-Mode plot produced by the DSS-8, DSS-3, and DES data were examined. Table 4 depicts the means and standard deviations of the base rate estimates produced by each of the three taxometric procedures. Inspection of the base rate estimates revealed that the majority of these estimates were substantially higher than would be theoretically expected on the basis of the documented relation between DS and the prevalence of various forms of associated psychopathology. Furthermore, the base rate estimates demonstrated considerable variability. Thus, when these base rate estimates are considered in combination with the consistently dimensional-shaped plots produced by several mathematically distinct taxometric procedures, the results of the present study are strongly supportive of dimensional structure. 
Table 4

Means (and Standard Deviations) of the Base Rate Estimates

Generated by the Disgust Sensitivity Scale and Disgust Emotion

Scale Data

\begin{tabular}{lccc}
\hline Indicator & MAXEIG & MAMBAC & L-mode \\
\hline DSS-8 & $.47(.12)$ & $.54(.10)$ & .51 \\
DSS-3 & $.52(.17)$ & $.54(.04)$ & .51 \\
DES & $.18(.04)$ & $.46(.05)$ & .50 \\
\hline
\end{tabular}

Note. MAXEIG $=$ maximum eigenvalue; $\mathrm{MAMBAC}=$ mean above minus below a cut; L-mode $=$ latent mode factor analysis; DSS-8 = Disgust Sensitivity Scale-Eight Indicators; DSS-3 = Disgust Sensitivity Scale-Three Indicators; DES = Disgust Emotion Scale.

\section{Discussion}

Researchers have begun to implicate DS as a potential risk factor for a range of anxiety and related disorders, including spider phobia (de Jong et al., 2002), BII phobia (Olatunji, Lohr, Sawchuk, \& Westendorf, 2005; Page, 2003), contamination-based OCD (Olatunji et al., 2004), health anxiety and hypochondriasis (Davey \& Bond, 2006), and eating disorders (Troop et al., 2002). DS is a genetically based personality trait that may operate as a specific risk for anxiety disorders in a similar fashion as anxiety sensitivity (i.e., Muris, 2006). Recent taxometric research has begun to examine the structure of anxiety sensitivity, with findings from several published studies appearing suggestive of taxonic latent structure (e.g., Bernstein, Zvolensky, Stewart, et al., 2006; Bernstein et al., 2005). However, the latent structure of DS has yet to be examined in a similar fashion. The absence of data directly speaking to the underlying latent structure of DS is somewhat concerning, as many studies have operated under the assumption that DS is either dimensional (i.e., Haidt et al., 1994; Olatunji, Williams, et al., 2005) or categorical (i.e., Schienle et al., 2001), with no empirical evidence to inform researchers on which approach is more appropriate for the assessment of DS given the nature of the construct. The present investigation uniquely addressed the paucity of empirical evidence that bears directly on the latent continuity of DS by conducting a taxometric analysis in two independent samples using two different measures of the DS construct.

The first sample of participants consisted of those who completed the DSS, the most widely used measure of DS in the research literature (Olatunji \& Sawchuk, 2005). We then examined the latent structure of DS, as assessed by the DSS, by applying three taxometric proceduresMAXEIG, MAMBAC, and L-Mode-to two sets of DSS indicators. Regardless of DSS indicator set, none of these procedures produced evidence for a latent DS taxon. Rather, results of the three procedures converged around a dimensional solution for DS, as indicated by characteristically dimensional plot shapes and base rate estimates that were highly discrepant from the prevalence of DS in the respective populations. 
Results of the present taxometric analysis of the DSS provide initial support for the dimensional nature of DS. It appears, therefore, that the heterogeneity of DS is best conceptualized as reflecting quantitative rather than qualitative differences among individuals (i.e., Gibb, Alloy, Abramson, Beevers, \& Miller, 2004). It has been noted that exclusive reliance on any one measure of a given construct in taxometric analysis is limiting (i.e., Bernstein, Zvolensky, Kotov, et al., 2006). Indeed, the failure to demonstrate a latent categorical structure with the DSS indicators does not necessarily prove that there is no boundary to be found. It is always possible that DS assessed by a different instrument or a different set of indicators might reveal a critical discontinuity. To address this issue, in the present study we also conducted taxometric analyses on a second sample of participants who completed the DES, a measure of DS developed to address some of the psychometric limitations (i.e., subscale reliability) of the DSS (Olatunji, Sawchuk, de Jong, \& Lohr, 2007). Results of the taxometric analysis with the DES were nearly identical to those with the DSS and suggestive of a latent DS dimension. Thus, DS appears to occur in various degrees and does not demarcate a discrete taxon.

These taxometric findings have important implications for the assessment of DS. First, a dimensional solution indicates that the use of continuous measures focused on evaluating the full range of DS will maximize statistical power and minimize information loss. Researchers should exercise caution in dichotomizing individuals on the basis of their scores on measures of DS, as any cutpoint would be arbitrary given the dimensional nature of DS. Second, a dimensional solution for DS suggests that additive and graded etiological models are most appropriate for pathological disgust reactions, rather than models that suggest a specific all-ornone genetic factor or a single environmental variable (Haslam, 1997). Thus, it is likely that various environment-related and person-specific factors are important in predicting the intensity and duration of an individual's disgust reactions and therefore should be specifically and systematically addressed in the assessment and treatment of disorders in which DS has been implicated. The dimensional results of the present study are also consistent with several recent theories regarding the etiology of DS. For example, in his analysis of the fear of contamination commonly observed in OCD, Rachman (2006) noted that DS may originate from the influence of a biologically based sensitivity (person-specific factor) that is manifested during exposures to the threat of contamination (environment-related factor) and is then consolidated or not, depending on the outcome (environment-related factor) of the exposures and the person's interpretation (person-specific factor) of these events.

These findings also have important implications for the assessment of DS in the clinical context. Recent work has highlighted the need to consider a shift to a dimensional classification of specific psychiatric conditions (Widiger \& Trull, 2007). These findings complement prior research suggesting that the psychological vulnerabilities that facilitate the development of specific psychiatric conditions should also be conceptualized and assessed along a dimensional continuum (Gibb et al., 2004). The implications of a dimensional latent structure also relate to the methods by which DS is identified and assessed in the context of preventing the onset of disorders in which DS has been implicated as a risk factor. Accurate measurement is the cornerstone of any intervention program geared toward prevention, and explication of the latent structure of DS may facilitate this process. The dimensional latent structure of DS suggests that 
focusing treatment on clinical cases above the threshold would miss subthreshold cases who are not qualitatively different and who may also benefit from intervention.

These dimensional findings regarding the latent structure of DS may also inform our understanding of the mechanisms that result in change in this variable. DS appears to be relatively unaffected by treatment, at least in individuals with spider phobia (de Jong, Andrea, \& Muris, 1997; de Jong, Vorage, \& van den Hout, 2000). However, very little is known about the processes that underlie the resistance of DS to extinction. It may be informative to assess DS in the context of its dimensional taxometric properties during clinical intervention to better understand that nature of change-related processes (or lack thereof). This future line of research should be informed by the present findings of a dimensional latent structure of DS, which implies that therapeutic change is best thought of as a gradual decline and not as a distinct change from pathology to normality. Accordingly, clinical assessment during treatment outcome research may be maximized by sampling of DS during multiple time points.

The current taxometric study exhibited a number of strengths, such as the inclusion of large samples, the use of multiple measures of the DS construct, and the use of multiple taxometric procedures to evaluate the replicability of the results. However, inferences on the basis of these findings should be made in the context of the study limitations. Indeed, one limitation of this study is that the search for taxonicity of DS was limited by the indicators that were chosen to represent it (Widiger, 2001). Although the indicators that were chosen were based on empirically informed studies of the psychometric properties of the DSS and DES, it is possible that the dimensional results were due, in part, to the DS indicators chosen. Future investigations would benefit from replication of the present findings with alternative measures of DS (i.e., the Disgust Propensity and Sensitivity Scale-Revised; van Overveld, de Jong, Peters, Cavanagh, \& Davey, 2006) as well as testing of additional manifest indicators of DS derived from alternative methods. Such methods may target DS as assessed by behavioral avoidance (Olatunji, Lohr, Sawchuk, \& Tolin, 2007), information processing (Charash \& McKay, 2002), or psychophysiology (Stark, Walter, Schienle, \& Vaitl, 2005). Such a multimethod approach to manifest indicators may provide further validation of dimensionality as well as promote psychometric refinement of the assessment of DS as it relates to anxiety and related disorders.

Another potential limitation of the present study was the exclusive use of an undergraduate sample, which may limit the generalizability of the taxometric findings. Although it is theoretically important to evaluate the latent structure of DS in clinical samples, it is equally important to examine it in nonclinical samples to determine which approach (categorical vs. dimensional) is appropriate for assessing individuals who may be at risk for developing anxiety and related problems. Although examination of the latent structure of DS in the context of a restricted range (i.e., only clinical samples) could limit the inferences that can be drawn, it could be argued that these dimensional findings were due to the use of nonclinical participants whose DS levels were not particularly high. However, this is unlikely, as the present samples evidenced a wide degree of DS, including participants whose DS levels would be considered in the clinical range. For example, Woody and Tolin (2002) reported a mean of $22.12(S D=4.86)$ on the DSS for patients with contamination-based OCD, and approximately $21 \%$ of our sample was above this mean. Furthermore, Olatunji, Lohr, Willems, and Sawchuk (2006) reported a mean of 62.43 (SD 
$=17.84$ ) on the DES for participants scoring in the clinical range on a measure of contamination-based OCD, and approximately $17 \%$ of our sample was above this mean. Therefore, it appears unlikely that the current results were due to the exclusive reliance on nonclinical DS data.

In conclusion, the present study used taxometrics to examine the latent structure of DS. Multiple taxometric procedures provided convergent evidence supporting a dimensional conceptualization of DS, indicating that DS is present to a greater or lesser degree in all individuals. An important area for future research is to examine whether there is a point on the continuum at which the strength of the relationship between DS and symptoms of specific disorders is significantly stronger. Although this study was thorough in its analyses, it is, to the best of our knowledge, the first taxometric analysis of DS, and thus our dimensional findings require replication in multiple contexts. For example, previous research has consistently observed that women report significantly higher levels of DS than men (Olatunji, Sawchuk, Arrindell, \& Lohr, 2005). Although it is possible that gender may not qualitatively change DS, future studies with sufficiently large independent samples for meaningful comparisons might explore the extent to which the taxometrics of DS vary as a function of gender. Operating under the assumption that DS is, indeed, a universal risk factor for certain anxiety and related disorders, future studies might also extend the taxometric analysis of DS to diverse crosscultural populations. In addition to informing our understanding of the nature of DS between cultures, future research along these lines may also have important theoretical and clinical implications for anxiety and related disorders in which DS has been implicated. 


\section{FOOTNOTES}

1. Two of the original DSS-8 indicators (representing the Sex and Hygiene subscales) did not meet the minimum validity criteria (one standard deviation). These indicators were dropped, and taxometric analyses were rerun. Results were nearly identical to those that included these two indicators. In addition, taxometric analyses were run both with and without the inclusion of the indicator associated with the Sympathetic Magic subscale of the DSS because of the questionable association between sympathetic magic and DS. Again, results were nearly identical to those that included all eight indicators. Thus, to reduce confusion and ease interpretability of findings, all figures and tables include all eight of the original DSS indicators.

2. We both examined and rated plots. In addition, three external consultants, who were experienced taxometricians, were recruited to evaluate the shape of the plots. Raters were also provided with a copy of the simulated dimensional and taxonic plots to assist in their ratings. It should be noted that not all of the MAMBAC taxonic simulations evidenced clear peaks, and thus, even though the simulated taxonic and dimensional plots were clearly distinguishable, raters were encouraged to exercise caution in using MAMBAC simulations to aid in their interpretation of the MAMBAC plots. All five examiners were in perfect agreement in rating the MAXEIG, MAMBAC, and L-Mode plots produced by the DSS-8, DSS-3, and DES data sets as indicative of a latent dimension.

\section{REFERENCES}

Beach, S. R. H., Amir, N., \& Bau, J. J. (2005). Can sample-specific simulations help detect low base-rate taxonicity?Psychological Assessment, 17, 446-461.

Bernstein, A., Zvolensky, M. J., Kotov, R., Arrindell, W. A., Taylor, S., Sandin, B., et al. (2006). Taxonicity of anxiety sensitivity: A multi-national analysis. Journal of Anxiety Disorders, 20, 122.

Bernstein, A., Zvolensky, M. J., Norton, P. J., Schmidt, N. B., Taylor, S., Forsyth, J. P., et al. (2007). Taxometric and factor analytic models of anxiety sensitivity: Integrating approaches to latent structural research. Psychological Assessment, 19, 74-87.

Bernstein, A., Zvolensky, M. J., Stewart, S. H., Comeau, M. N., \& Leen-Feldner, E. W. (2006). Anxiety sensitivity taxonicity across gender among youth. Behaviour Research and Therapy, 44, 679-698.

Bernstein, A., Zvolensky, M. J., Weems, C., Stickle, T., \& Leen-Feldner, E. (2005). Taxonicity of anxiety sensitivity: An empirical test among youth. Behaviour Research and Therapy, 43, 11311155.

Björklund, F., \& Hursti, T. J. (2004). A Swedish translation and validation of the Disgust Scale: A measure of disgust sensitivity. Scandinavian Journal of Psychology, 45, 279-284. 
Broman-Fulks, J. J., Ruggiero, K. J., Kilpatrick, D. G., Resnick, H. S., Green, B. A., \& Saunders, B. E. (2006). Taxometric investigation of posttraumatic stress disorder: Data from two nationally representative samples. Behavior Therapy, 37, 364-380.

Charash, M., \& McKay, D. (2002). Attention bias for disgust. Journal of Anxiety Disorders, 16, 529-541.

Cisler, J., Reardon, J., Williams, N., \& Lohr, J. M. (2007). Anxiety sensitivity and disgust sensitivity interact to predict contamination fears. Personality and Individual Differences, 42, 935-946.

Davey, G. C., L. \& Bond, N. (2006). Using controlled comparisons in disgust psychopathology research: The case of disgust, hypochondriasis and health anxiety. Journal of Behavior Therapy and Experimental Psychiatry, 37, 4-15.

Davey, G. C. L., Buckland, G., Tantow, B., \& Dallos, R. (1998). Disgust and eating disorders. European Eating Disorders Review, 6, 201-211.

de Jong, P. J., Andrea, H., \& Muris, P. (1997). Spider phobia in children: Disgust and fear before and after treatment. Behaviour Research and Therapy, 35, 559-562.

de Jong, P. J., \& Merckelbach, H. (1998). Blood-injection-injury phobia and fear of spiders: Domain specific individual differences in disgust sensitivity. Personality and Individual Differences, 24, 153-158.

de Jong, P. J., \& Muris, P. (2002). Spider phobia: Interaction of disgust and perceived likelihood of involuntary physical contact. Journal of Anxiety Disorders, 16, 51-65.

de Jong, P. J., Peters, M., \& Vanderhallen, I. (2002). Disgust and disgust sensitivity in spider phobia: Facial EMG in response to spider and oral disgust imagery. Journal of Anxiety Disorders, 16, 477-493.

de Jong, P. J., Vorage, I., \& van den Hout, M. A. (2000). Counterconditioning in the treatment of spider phobia: Effects on disgust, fear and valence. Behaviour Research and Therapy, 38, 1055-1069.

Gibb, B. E., Alloy, L. B., Abramson, L. Y., Beevers, C. G., \& Miller, I. W. (2004). Cognitive vulnerability to depression: A taxometric analysis. Journal of Abnormal Psychology, 113, 81-89.

Haidt, J., McCauley, C., \& Rozin, P. (1994). Individual differences in sensitivity to disgust: A scale sampling seven domains of disgust elicitors. Personality and Individual Differences, 16, 701-713.

Haslam, N. (1997). Evidence that male sexual orientation is a matter of degree. Journal of Personality and Social Psychology, 73, 862-870.

Haslam, N. (2003). Categorical versus dimensional models of mental disorder: The taxometric evidence. Australian and New Zealand Journal of Psychiatry, 37, 696-704. 
Husted, D., Shapira, N., \& Goodman, W. (2006). The neurocircuitry of obsessive-compulsive disorder and disgust. Progress in Neuro-Psychopharmacology and Biological Psychiatry, 30 , 389-399.

Kleinknecht, R. A., Kleinknecht, E. E., \& Thorndike, R. M. (1997). The role of disgust and fear in blood and injection-related fainting symptoms: A structural equation model. Behaviour Research and Therapy, 35, 1075-1087.

Kotov, R., Schmidt, N. B., Lerew, D. R., \& Joiner, T. E. (2005). Latent structure of anxiety: Taxometric exploration. Psychological Assessment, 17, 369-374.

Lenzenweger, M. F., \& Korfine, L. (1992). Confirming the latent structure and base rate of schizotypy: A taxometric analysis. Journal of Abnormal Psychology, 101, 567-571.

Mancini, F., Gragnani, A., \& D'Olimpio, F. (2001). The connection between disgust and obsessions and compulsions in a non-clinical sample. Personality and Individual Differences, 31, 1173-1180.

Marzillier, S. L., \& Davey, G. C. L. (2004). The emotional profiling of disgust-eliciting stimuli: Evidence for primary and complex disgusts. Cognition \& Emotion, 18, 313-336.

Matchett, G., \& Davey, G. C. (1991). A test of a disease-avoidance model of animal phobias. Behaviour Research and Therapy, 29, 91-94.

McNally, R. J. (2002). Anxiety sensitivity and panic disorder. Biological Psychiatry, 52, 938-946.

Meehl, P. E. (1973). MAXCOV-HITMAX: A taxonomic search method for loose genetic syndromes. In P. E.Meehl (Ed.), Psychodiagnosis: Selected papers (pp. 200-224). Minneapolis: University of Minnesota Press.

Meehl, P. E. (1995). Bootstraps taxometrics: Solving the classification problem in psychopathology. American Psychologist, 50, 266-275.

Meehl, P. E., \& Golden, R. R. (1982). Taxometric methods. In J. N.Butcher \& P. C.Kendall (Eds.), The handbook of research methods in clinical psychology (pp. 127-181). New York: Wiley.

Meehl, P. E., \& Yonce, L. J. (1994). Taxometric analysis: I. Detecting taxonicity with two quantitative indicators using means above and below a sliding cut (MAMBAC procedure). Psychological Reports, 74, 1059-1274.

Meehl, P. E., \& Yonce, L. J. (1996). Taxometric analysis: II. Detecting taxonicity using covariance of two quantitative indicators in successive intervals of a third indicator (MAXCOV procedure). Psychological Reports, 78, 1091-1227.

Merckelbach, H., de Jong, P., Arntz, A., \& Schouten, E. (1993). The role of evaluative learning and disgust sensitivity in the etiology and treatment of spider phobia. Advances in Behaviour Research and Therapy, 15, 243-255. 
Mulkens, S. A., de Jong, P. J., \& Merckelbach, H. (1996). Disgust and spider phobia. Journal of Abnormal Psychology, 105, 464-468.

Muris, P. (2006). The pathogenesis of childhood anxiety disorders: Considerations from a developmental psychopathology perspective. International Journal of Behavioral Development, 30, 5-11.

Muris, P., Merckelbach, H., Schmidt, H., \& Tierney, S. (1999). Disgust sensitivity, trait anxiety and anxiety disorders symptoms in normal children. Behaviour Research and Therapy, 37, 953961.

Olatunji, B. O. (2006). Evaluative learning and emotional responding to fearful and disgusting stimuli in spider phobia. Journal of Anxiety Disorders, 20, 858-876.

Olatunji, B. O., Arrindell, W., \& Lohr, J. M. (2005). Can the sex differences in disgust sensitivity account for the sex differences in blood-injection-injury fears?Personality and Individual Differences, 39, 61-71.

Olatunji, B. O., Forsyth, J. P., \& Cherian, A. (2007). Evaluative conditioning of disgust: A sticky form of relational learning that is resistant to extinction. Journal of Anxiety Disorders, 21, 820834.

Olatunji, B. O., Lohr, J. M., Sawchuk, C. N., \& Tolin, D. F. (2007). Multimodal assessment of disgust in contamination-related obsessive compulsive disorder. Behaviour Research and Therapy, 45, 263-276.

Olatunji, B. O., Lohr, J. M., Sawchuk, C. N., \& Westendorf, D. (2005). Using facial expressions as CSs and fearsome and disgusting pictures as UCSs: Affective responding and evaluative learning of fear and disgust in BII phobia. Journal of Anxiety Disorders, 19, 539-555.

Olatunji, B. O., Lohr, J. M., Willems, J. L., \& Sawchuk, C. N. (2006). Expectancy bias for disgust and emotional responding in contamination-related obsessive-compulsive disorder. Anxiety, Stress, and Coping, 19, 383-396.

Olatunji, B. O., \& Sawchuk, C. N. (2005). Disgust: Characteristic features, social implications, and clinical manifestations. Journal of Social and Clinical Psychology, 24, 932-962.

Olatunji, B. O., Sawchuk, C. N., Arrindell, W. A., \& Lohr, J. M. (2005). Disgust sensitivity as a mediator of the sex differences in contamination fears. Personality and Individual Differences, $38,713-722$.

Olatunji, B. O., Sawchuk, C. N., de Jong, P. J., \& Lohr, J. M. (2006). The structural relation between disgust sensitivity and blood-injection-injury fears: A cross-cultural comparison of U.S. and Dutch data. Journal of Behavior Therapy and Experimental Psychiatry, 37, 16-29.

Olatunji, B. O., Sawchuk, C. N., de Jong, P. J., \& Lohr, J. M. (2007). Disgust sensitivity and anxiety disorder symptoms: Psychometric properties of the Disgust Emotions Scale. Journal of Psychopathology and Behavioral Assessment, 29, 115-124. 
Olatunji, B. O., Sawchuk, C. N., Lohr, J. M., \& de Jong, P. J. (2004). Disgust domains in the prediction of contamination fear. Behaviour Research and Therapy, 42, 93-104.

Olatunji, B. O., Sawchuk, C. N., Lohr, J. M., \& Patten, K. (2007). Fear and disgust responding to heterogeneous blood-injection-injury stimuli. Journal of Psychopathology and Behavioral Assessment, 29, 1-8.

Olatunji, B. O., Tolin, D. F., Huppert, J., \& Lohr, J. M. (2005). The relation between fearfulness, disgust sensitivity and religious obsessions in a non-clinical sample. Personality and Individual Differences, 38, 891-902.

Olatunji, B. O., Williams, N. L., Lohr, J. M., \& Sawchuk, C. N. (2005). The structure of disgust: Domain specificity in relation to contamination ideation and excessive washing. Behaviour Research and Therapy, 43, 1069-1086.

Olatunji, B. O., Williams, N. L., Sawchuk, C. N., \& Lohr, J. M. (2006). Disgust, anxiety, and fainting symptoms in blood-injection-injury fears: A structural model. Journal of Anxiety Disorders, 20, 23-41.

Page, A. C. (2003). The role of disgust in faintness elicited by blood and injection stimuli. Journal of Anxiety Disorders, 17, 45-58.

Phillips, M. L., Senior, C., Fahy, T., \& David, A. S. (1998). Disgust-the forgotten emotion of psychiatry. British Journal of Psychiatry, 173, 373-375.

Rachman, S. (2006). Fear of contamination: Assessment and treatment. New York: Oxford University Press.

Reiss, S., \& McNally, R. J. (1985). Expectancy model of fear. In S.Reiss \& R. R.Bootzin (Eds.), Theoretical issues in behavior therapy (pp. 107-121). San Diego, CA: Academic Press.

Rozin, P., \& Fallon, A. E. (1987). A perspective on disgust. Psychological Review, 94, 23-41.

Rozin, P., Haidt, J., \& McCauley, C. R. (2000). Disgust. In M.Lewis \& J. M.Haviland (Eds.), Handbook of emotions (pp. 575-594). New York: Guilford Press.

Ruscio, A. M., Borkovec, T. D., \& Ruscio, J. (2001). A taxometric investigation of the latent structure of worry. Journal of Abnormal Psychology, 110, 413-422.

Ruscio, J. (2006). Documentation of program code to perform taxometric analyses and simulate comparison data in the $R$ language. Retrieved November 1,2006 , from http://www.taxometricmethod.com/TaxProg\%206-9-07.R

Ruscio, J. (2007). Taxometric programs for the R computing environment: User's manual. Retrieved from http://www.taxometricmethod.com/TaxProgManual\%207-5-07.pdf

Ruscio, J., Haslam, N., \& Ruscio, A. M. (2006). Introduction to the taxometric method: A practical guide. Mahwah, NJ: Erlbaum. 
Ruscio, J., \& Marcus, D. K. (2007). Detecting small taxa using simulated comparison data: A reanalysis of Beach, Amir, and Bau's (2005) data. Psychological Assessment, 19, 241-246.

Ruscio, J., Ruscio, A. M., \& Meron, M. (2007). Applying the bootstrap to taxometric analysis: Generating empirical sampling distributions to help interpret results. Multivariate Behavioral Research, 42, 349-386.

Sawchuk, C. N., Lohr, J. M., Lee, T. C., \& Tolin, D. F. (1999). Exposure to disgust-evoking imagery and information processing biases in blood-injection-injury phobia. Behaviour Research and Therapy, 37, 249-257.

Sawchuk, C. N., Lohr, J. M., Tolin, D. F., Lee, T. C., \& Kleinknecht, R. A. (2000). Disgust sensitivity and contamination fears in spider and blood-injection-injury phobias. Behaviour Research and Therapy, 38, 753-762.

Sawchuk, C. N., Lohr, J. M., Westendorf, D. H., Meunier, S. A., \& Tolin, D. F. (2002). Emotional responding to fearful and disgusting stimuli in specific phobics. Behaviour Research and Therapy, 40, 1031-1046.

Schienle, A., Stark, R., \& Vaitl, D. (2001). Evaluative conditioning: A possible explanation for the acquisition of disgust responses?Learning and Motivation, 32, 65-83.

Schienle, A., Stark, R., Walter, B., \& Vaitl, D. (2003). The connection between disgust sensitivity and blood-related fears, faintness symptoms, and obsessive-compulsiveness in a non-clinical sample. Anxiety, Stress, and Coping, 16, 185-193.

Schmidt, N. B., Kotov, R., Lerew, D. R., Joiner, T. E., \& Ialongo, N. S. (2005). Evaluating latent discontinuity in cognitive vulnerability to panic: A taxometric investigation. Cognitive Therapy and Research, 29, 673-690.

Stark, R., Walter, B., Schienle, A., \& Vaitl, D. (2005). Psychophysiological correlates of disgust and disgust sensitivity. Journal of Psychophysiology, 19, 50-60.

Thorpe, S. J., Patel, S. P., \& Simonds, L. M. (2003). The relationship between disgust sensitivity, anxiety and obsessions. Behaviour Research and Therapy, 41, 1397-1409.

Thorpe, S. J., \& Salkovskis, P. M. (1998). Studies on the role of disgust in the acquisition and maintenance of specific phobias. Behaviour Research and Therapy, 36, 877-893.

Tolin, D. F., Lohr, J. M., Sawchuk, C. N., \& Lee, T. C. (1997). Disgust and disgust sensitivity in blood-injection-injury and spider phobia. Behaviour Research and Therapy, 35, 949-953.

Tolin, D. F., Woods, C. M., \& Abramowitz, J. S. (2006). Disgust sensitivity and obsessivecompulsive symptoms in a nonclinical sample. Journal of Behavior Therapy and Experimental Psychiatry, 37, 30-40.

Troop, N. A., Murphy, F., Bramon, E., \& Treasure, J. L. (2000). Disgust sensitivity in eating disorders: A preliminary investigation. International Journal of Eating Disorders, 27, 446-451. 
Troop, N. A., Treasure, J. L., \& Serpell, L. (2002). A further exploration of disgust in eating disorders. European Eating Disorders Review, 10, 218-226.

van Overveld, M., de Jong, P. J., Peters, M. L., Cavanagh, K., \& Davey, G. C. L. (2006). Disgust propensity and disgust sensitivity: Separate constructs that are differentially related to specific fears. Personality and Individual Differences, 41, 1241-1252.

Waller, N. G., \& Meehl, P. E. (1998). Multivariate taxometric procedures: Distinguishing types from continua. Newbury Park, CA: Sage.

Widiger, T. A. (2001). What can be learned from taxometric analyses. Clinical Psychology: Science and Practice, 8, 528-533.

Widiger, T., A. \& Trull, T. J. (2007). Plate tectonics in the classification of personality disorder: Shifting to a dimensional model. American Psychologist, 62, 71-83.

Woody, S. R., McLean, C., \& Klassen, T. (2005). Disgust as a motivator of avoidance of spiders. Journal of Anxiety Disorders, 19, 461-475.

Woody, S. R., \& Teachman, B. A. (2000). Intersection of disgust and fear: Normative and pathological views. Clinical Psychology: Science and Practice, 7, 291-311.

Woody, S. R., \& Tolin, D. F. (2002). The relationship between disgust sensitivity and avoidant behavior: Studies of clinical and nonclinical samples. Journal of Anxiety Disorders, 16, 543-559. 